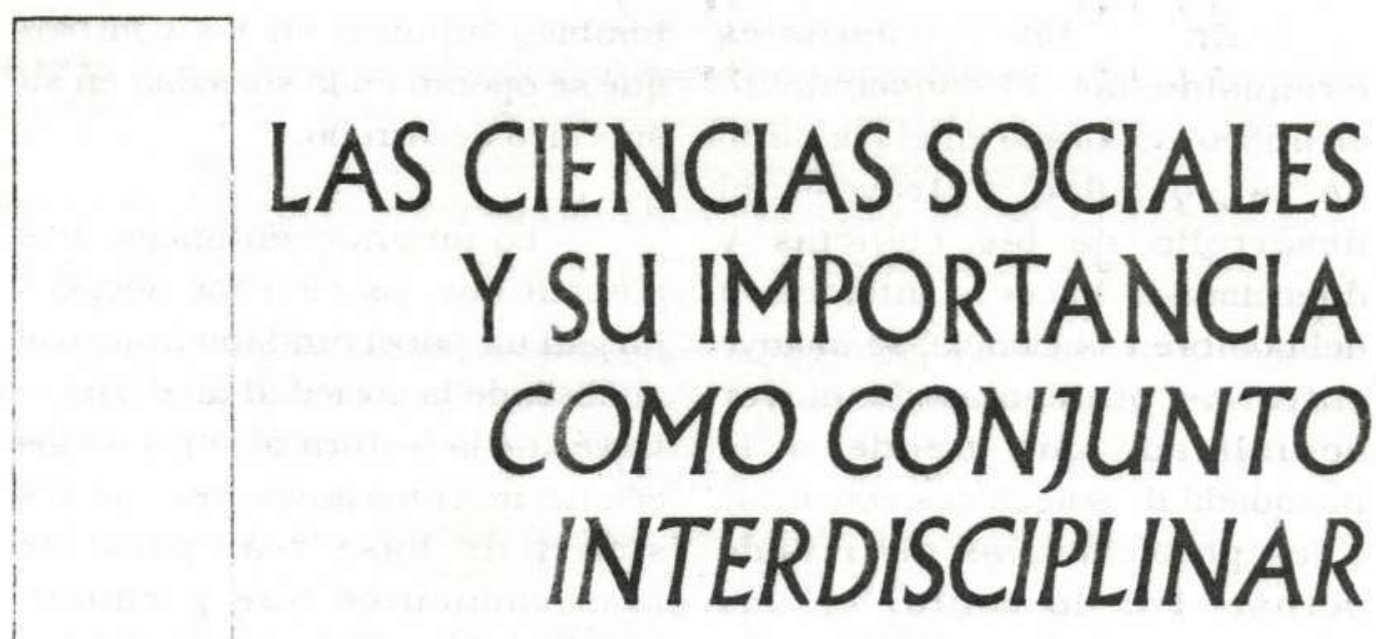

RICARDO A. CASTAÑO Z. Ph. D. en Historia Profesor Universidad Autónoma de Manizales

A n f o r a

I rasgo caracteristico de la epoca actual es de cambios rardinales cada vez mas acelerados. relacionados con lodas las esferas de la vida y fundamentalmente. ron la actividad del hombre en las nuevas sociedades. Estas transformaciones tienen que ver con el proceso de construcción de nuevos esquemas de desarrollo económico o modelos alternativos de desarrollo. nuevas modalidades de hacer política y por ende. nuevas interpretaciones de la acción del hombre en la sociedad a instancias de la gran revolución científico-técnica. Se operan cambios, entonces, en las condiciones objetivas de la existencia y la convivencia del hombre. en su vida espiritual y también en su actitud frente a la realidad circundante. 
En las actuales circunstancias, el conocimiento científico elaborado en el conjunto de la sociedad a través del desarrollo de las ciencias y direccionado hacia la interacción del hombre y la ciencia, se incluye entre los problemas de mayor actualidad, que tiende a la búsqueda de soluciones concretas a las problemáticas del mundo actual. Por lo tanto, en las condiciones actuales de desarrollo de la ciencia y la tecnología, adquiere c a p it a 1 importancia. no la influencia q $u$ e ejercen los cambios sociales sobre las ciencias

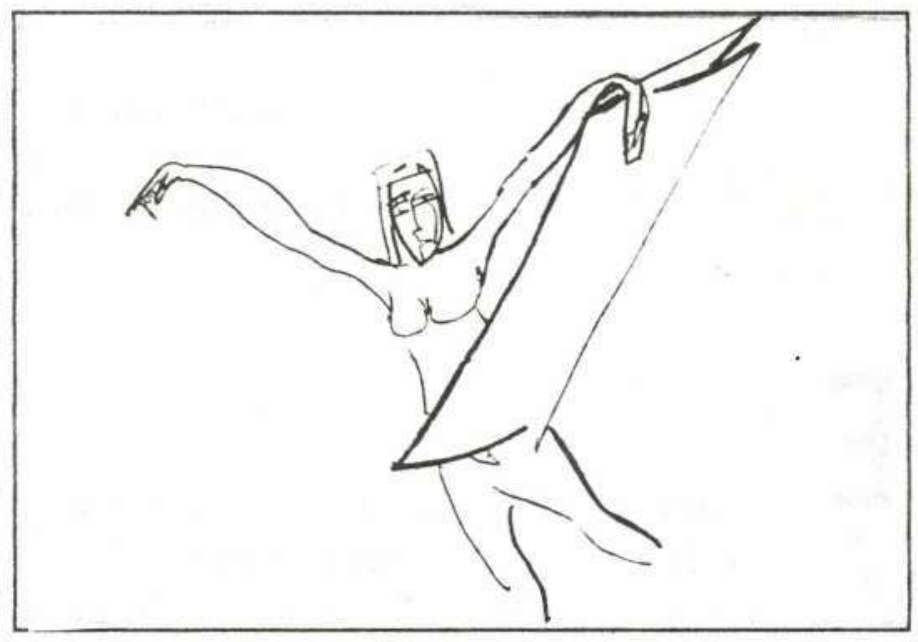

Para el hombre, el conocimiento de la sociedad ha sido $\mathrm{m} \mathrm{u} \mathrm{c} \mathrm{ho}$ más difícil de obtener que el relativo al universo material sociales, sino la capacidad de éstas de plantear soluciones a las problemáticas que se presentan.

\section{En el mundo} contemporáneo, la ciencia y la sociedad tienen una relación de reciprocidad. De igual manera como al interior de las ciencias se producen cambios debido a las transformaciones sociales, éstas que lo rodea y "precisamente porque las ciencias sociales estudian las fuentes de la acción social humana tienen una relación más inmediata con la historia que las ciencias naturales. Esta es la razón de que se deba dar una explicación del lugar que ocupan las ciencias sociales en cualquier intento amplio de dar cuenta del desarrollo de la ciencia en general 
en relación con el desarrollo de la sociedad». (1)

En este sentido el estudio del desarrollo de la sociedad es un imperante a instancias del cual podemos realizar un análisis serio de nuestro pasado sin caer en posiciones tendenciosas y antiéticas.

Existen. según Cardoso, cuatro maneras diferentes de abordar las relaciones e $\mathrm{n} \mathrm{t} r$ e ciencia y su relación con la sociedad. La primera de tipo determinista que se ciñe. únicamente. a los resultados

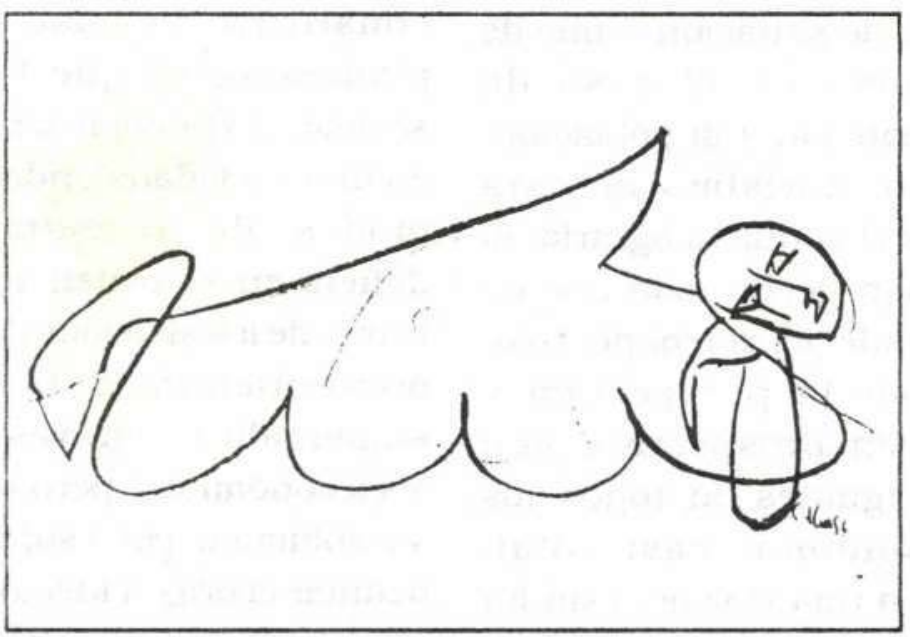

como reflejos de los factores dinámicos de tipo económico-social. afirma que todas las teorías científicas deben explicarse desde el contexto histórico en que surgen. sin dar lugar a discusión sobre su evolución en el tiempo.

La segunda, que señala que la ciencia determina lo social y es totalmente opuesta a la primera, lo
La tercera, plantea que lo científico y lo social son incompatibles por la complejidad misma de sus procesos y hace de
la ciencia una estructura ajena a
la sociedad, autorregulada y
cerrada. misma de sus procesos y hace de
la ciencia una estructura ajena a
la sociedad, autorregulada y
cerrada. misma de sus procesos y hace de
la ciencia una estructura ajena a
la sociedad, autorregulada y
cerrada. misma de sus procesos y hace de
la ciencia una estructura ajena a
la sociedad, autorregulada y
cerrada.

que implicaría que ésta tendría su propia dinámica interna, independiente, sin sufrir el impacto de lo social. Y la c u a r t a s e r i a mírar la ciencia, en $\mathrm{s}$ $\mathrm{u}$ autonomía relativa. e n interacdiones recíprocas con el conjunto de las estructuras de la sociedad. que es, tal vez, la más acertada y se acerca más a la realidad social actual y su relación con las ciencias de la sociedad. (2).

No podemos dejar de reconocer que las ciencias sociales han estado en una situación de atraso frente a los problemas sociales reales, que los científicos 


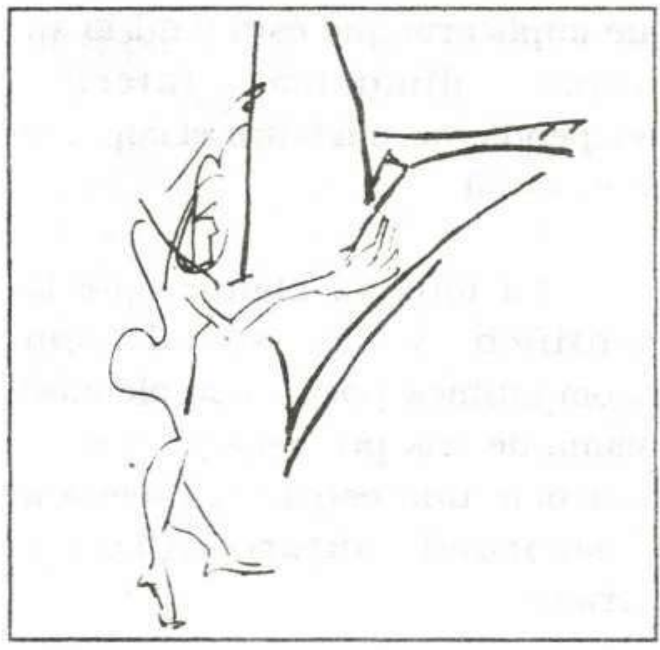

sociales recurren al análisis inmediatista de situaciones que de antaño, como en el caso de Colombia, aquejan a la población. Un ejemplo clarisimo de esta situación es el conflicto agrario, al cual se le han hecho todo tipo de análisis desde el retrospectivo. pasando por la perspectiva y mirándolo en prospectiva, con resultados iguales en todos los casos, abandono. casi total, conducente a una violencia sin fin en el laberinto de otra serie de conflictos sociales y económicos.

De lo anterior se desprende que si bien en otras ciencias es posible progresar a través de la construcción del conocimiento en su desarrollo teórico o experimental, en las ciencias sociales ésto es prácticamente imposible porque la velocidad de los cambios sociales contrasta con la lentitud con que se aborda el análisis de lo social, la realidad social es mucho más compleja que la realidad material, no responde directamente a relaciones causales $y$ es mucho más difícil de abordar en su conjunto como realidad objetiva, dado que implica procesos subjetivos, ideológicos e interpretativos.

Otro problema que ha atentado con frecuencia contra las ciencias sociales. y que obviamente constituye la base de lo aqui planteado, es que las ciencias sociales no pueden ser imparciales en una sociedad donde se imponen modos de pensamiento que defienden el orden imperante a través de los sistemas de educación. manteniendo a la población sumergida en grayes problemas socieconómicos, pero al margen de su solución. Por eso, no es difícil deducir el origen ideológico de esas ciencias que nacen al servicio del estado como disciplinas educativas para inculcar valores en defensa del estamento.

No debemos olvidar, además, que en muchas situaciones se recurre a la falsificación de hechos históricos los cuales se presentan como una historia acrítica en una sociedad conforme con los acontecimientos

\section{Anfóra}


políticos y económicos del momento, como sucediera, por ejemplo, con Chile.

Hoy las ciencias sociales se han constituido en una necesidad objetiva e irrefutable, ya que una sociedad analfabeta en ellas es altamente peligrosa y manipulable.

Como actividad científica del conocimiento las ciencias sociales aprehenden la realidad, primero, a partir de conjuntos teóricos relativos al movimiento de las sociedades $\mathrm{y}$, segundo, mediante actividades de investigación científica referentes a tales dinámicas sociales. Como actividad de conocimiento, son tanto una práctica social que busca incidir sobre un sistema determinado mediante la acción de crítica que proviene del conocimiento de las fuerzas o formas de cambio, como una práctica social específica del científico, pues tiene normas y reglas adecuadas para una relación explicativa de la realidad.

En tal sentido, es posible afirmar que las ciencias sociales actualmente presentan una doble tarea: por una parte explican e interpretan la realidad social, es decir, crean teorías, leyes y modelos que permiten comprender en general los movimientos sociales, posibilitan la comprensión de la dinámica social a partir del análisis de cada uno de los componentes de dicha realidad y de su integración como un todo interrelacionado multicausal y multivariado.

Por otra parte, permiten la construcción de modelos aplicables a la vida de las sociedad particulares y en tal sentido se constituyen en un aporte práctico para el desarrollo de la soctedad. permiten la solución de problemas concretos y la previsión de situaciones futuras orientadas al crecimiento y bienestar de la sociedad.

Ahora bien, cada una de las ciencias sociales aborda de manera particular y diferenciada aspectos parciales de la realidad social. Ninguna de ellas es autosuficiente por sí misma; las ciencias sociales

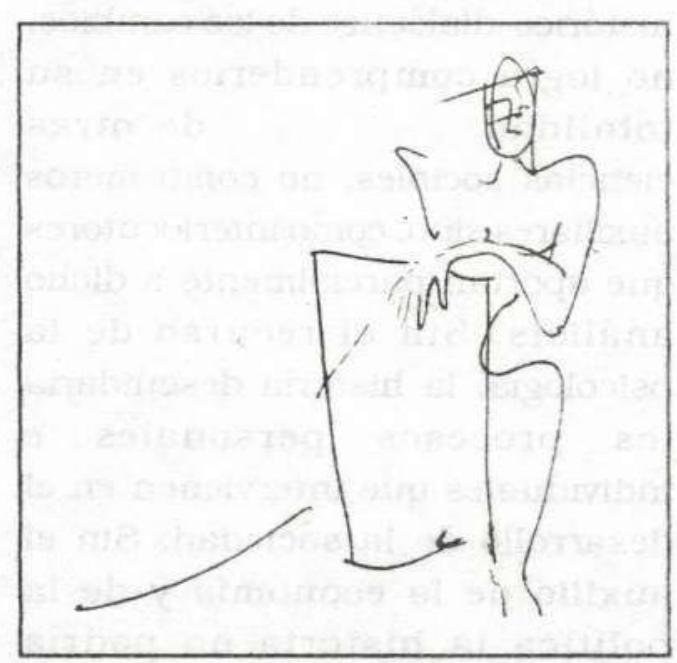


no sólo utilizan métodos y aportes de las ciencias naturales, sino, cada una de ellas complementa los aportes de las demás.

Así, para comprender en su totalidad un hecho social debe hacerse énfasis en el estudio de los procesos históricos que desembocan en los cambios actuales. No se debería insertar cosas nuevas dentro de las viejas en vías de extinción, sino se necesitaría realizar un análisis histórico-dialéctico de dichos cambios basado en lo que planteó Hegel: «Todo nacimiento y muerte, en lugar de ser una continuación paulatina, son por el contrario una interrupción de la misma y un salto del cambio cuantitativo al cualitativo» (3).

Sin embargo, la historia aunque permite el análisis histórico-dialéctico de los cambios, no logra comprenderlos en su totalidad.

de otras ciencias sociales, no como meros auxiliares sino, como interlocutores que aportan parcialmente a dicho análisis. Sin el recurso de la psicología, la historia descuidaría los procesos personales e individuales que intervienen en el desarrollo de la sociedad. Sin el auxilio de la economía y de la política la historia no podría interpretar las causales de dichos procesos, y sin la ayuda de la sociología no podría recomponer en su totalidad la realidad actual y comprender sus tendencias futuras.

Se trata, por lo tanto de mirar el proceso social como movimiento, que puede interpretarse como lo diría Engels «Hombres dotados de conciencia» que persiguen determinados fines; de analizar los acontecimientos en relación con otros, no aisladamente puesto que ningún fenómeno existe por sí solo, sino como elemento integrante de un proceso.

Ningún científico social está en capacidad de hacer un análisis lo suficientemente objetivo, si no tiene en cuenta la unidad del proceso general, en el que los

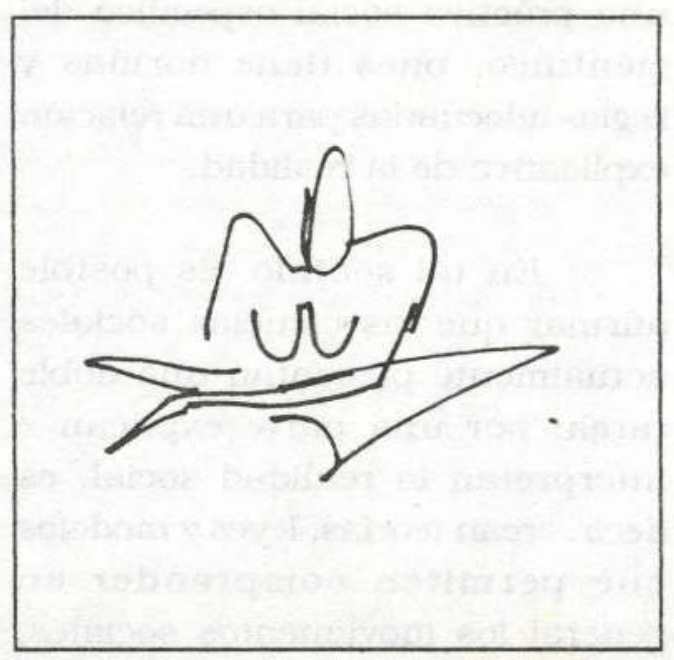


fenómenos por él analizados representan apenas parte del todo integrado. Así que toda absolutización de cualquier aspecto social, económico, político o histórico, conduce, sí duda, a su tergiversación.

Es cierto que ahora están en auge las tendencias a integrar y diferenciar las ciencias (en particular, las que estudian los diferentes problemas relacionados con el hombre y la sociedad). Pero cualquier integración del saber no es la fusión ni la dilución recíproca de las ciencias, sino la interacción y el enriquecimiento mutuo con miras a solucionar conjuntamente los problemas integrales. No pueden llegar a comprenderse los procesos de la vida ni el mundo del hombre, sin tomarse en cuenta los aspectos más disímiles de su realidad y sin incorporar al arsenal creador del científico todo lo logrado en las otras ramas del saber.

En las comunidades académicas y científicas sociales es común encontrar la discusión permanente sobre cuál de las ciencias sociales debe tener la primacía, cuál es la más importante, cuál es la que mayores aportes teóricos realiza y cuál es la que permite mayor desarrollo social. Estas discusiones sin

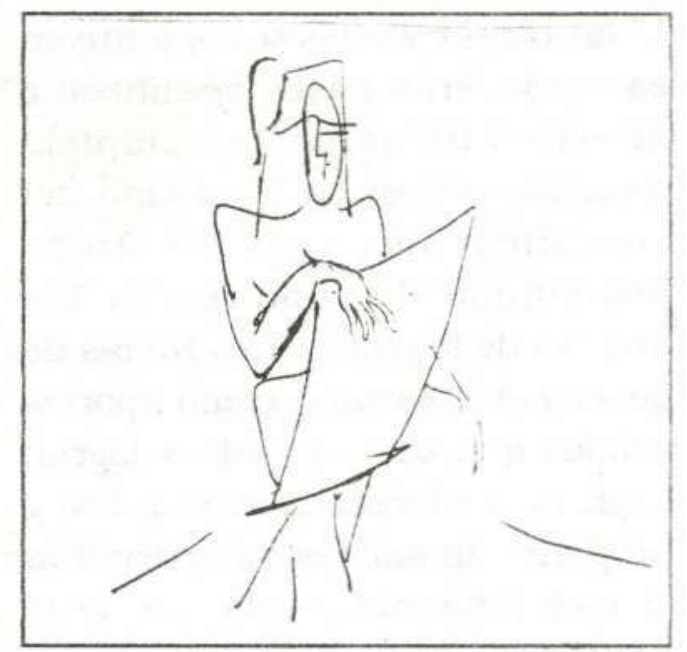

embargo poco aportan al desarrollo de la ciencia y, peor aún, dificultan el logro de objetivos comunes y aportes verdaderamente significativos para la sociedad.

El trabajo conjunto de las ciencias sociales es la única garantía de seguridad de que sus aportes sean verdaderamente valiosos tanto a nivel teórico, como a nivel práctico. No se trata simplemente de unir los resultados de las diferentes ciencias. Este remedo de interdisciplinariedad sólo logra dificultar el análisis del todo social.

La creación de verdaderos procesos interdisciplinarios, o más bien transdisciplinarios, se logra sólo cuando todas las ramas de las ciencias sociales permiten aportes parciales que unidos a los aportes 
de las otras ciencias se constituyen en verdaderas bases analíticas $e$ interpretativas de la compleja realidad social. La humildad del científico social es la única posibilidad de comprender los aportes de las diferentes ramas de las ciencias sociales como aportes válidos que unidos a otros logran explicar a interpretar la realidad y proponer dinámicas que permitan el mejoramiento paulatino de la sociedad.

\section{El} conjunto interdisciplinario de las ciencias sociales no garantiza su objetividad, no permite asegurar la verdad de sus predicados, pero se constituye en la única posibilidad de asegurar la construcción de conocimientos críticamente probados y seguramente útiles para el desarrollo de la sociedad.
(1) Bernal John D. Historia social de la ciencia TII De. Ciencias Sociales, La Habana 1986, pág. 238.

(2) Ciro F. S., Cardoso. Introducción al trabajo de la investigación histórica. Ed. Crítica. Barcelona 1987.pág 69-70.

(3) G. W. F. Hégel. La ciencia de la lógica. Berlín 1971, pág. 383.

\section{BIBLIOGRAFIA}

${ }^{*}$ G. W. F. Hegel. La ciencia de la lógica. Berlín 1971

*BERNAL, John. Historia social de la ciencia T II De. Ciencias Sociales La Habana 1986.

${ }^{*}$ CIRO S. Cardoșo. Introducción al trabajo de la investigación histórica. De Crítica. Barcelona 1987

*Ciencias Sociales Contemporáneas No. 9, 13. 11 . 